\title{
Clinical and treatment-related determinants of subjective quality of life in patients with first-episode psychosis
}

\author{
Vivian Wing Yan Kwong a, Wing Chung Chang a, b, *, Gloria Hoi Kei Chan a, Olivia Tsz Ting Jim a, \\ Emily Sin Kei Lau ${ }^{\text {a }}$, Christy Lai Ming Hui ${ }^{\text {a }}$, Sherry Kit Wa Chan ${ }^{\text {a, b }}$, Edwin Ho Ming Lee ${ }^{a}$, Eric Yu Hai Chen ${ }^{\text {a, b }}$ \\ a Department of Psychiatry, The University of Hong Kong, Queen Mary Hospital, Pokfulam, Hong Kong \\ ${ }^{\mathrm{b}}$ State Key Laboratory of Brain and Cognitive Sciences, the University of Hong Kong, Hong Kong
}

\section{A R T I C L E IN F O}

\section{Introduction}

Subjective quality of life (SQoL) has been regarded as an important outcome dimension in schizophrenia and related psychoses (Malla and Payne, 2005; Eack and Newhill, 2007). Although there is a lack of consensus definition, SQoL is generally conceptualized as a multi-dimensional construct which refers to an individual's perceived satisfaction with various life domains encompassing physical health, mental wellbeing and social functioning (Lehman, 1996; World Health Organization, 1998). It is acknowledged that one of the most fundamental features underlying SQoL construct is its subjective nature which provides an avenue to understand patients' life circumstances through their own perspectives (Lehman, 1996). Literature has indicated that schizophrenia is associated with reduced SQoL. A wide array of factors have been found to influence patients' self-appraisal of life satisfaction such as symptomatology, functional disability, different phases of illness, treatment characteristics and socio-cultural values, to name a few (Lehman, 1996; Karow et al., 2014). Accumulating evidence has shown that SQoL predicts symptomatic remission, relapse and functional outcome in schizophrenia (Lambert et al., 2007; Boyer et al., 2013a). Recent data has further suggested potential clinical utility of integrating SQoL assessment with feedback in routine psychiatric care for schizophrenia patients to enhance patient-clinician communication and their service satisfaction (Boyer et al., 2013b).

Recently, an increasing number of research has been conducted to examine SQoL in patients with first-episode psychosis (FEP) who were found to have lower levels of satisfaction across most SQoL domains than the general population (Law et al., 2005). Depression has consistently been found to be a robust predictor of SQoL in first-episode populations (Malla et al., 2004; Sim et al., 2004; Law et al., 2005; Melle et al., 2005, 2010; Wegener et al., 2005; Cotton et al., 2010; Renwick et al., 2012; Gardsjord et al., 2016). Discrepant findings, however, were observed with respect to the relationships between other clinical variables and SQoL in FEP, with some studies showing premorbid adjustment (Malla et al., 2004; Melle et al., 2005; MacBeth et al., 2015), duration of untreated psychosis (DUP) (Malla et al., 2004; Melle et al., 2005; Gorna et al., 2008), positive symptoms (Gorna et al., 2008; Chee, 2010; Cotton et al., 2010), negative symptoms (Malla et al., 2004; Gorna et al., 2008; Cotton et al., 2010; Thorup et al., 2010) and func-

Corresponding author at: Department of Psychiatry, The University of Hong Kong Queen Mary Hospital, Pokfulam, Hong Kong.

Email address: changwc@hku.hk (W.C. Chang) 
tioning (Cotton et al., 2010; Melle et al., 2010; Renwick et al., 2015) as independent factors associated with SQoL, but not others.

Of note, the majority of previous first-episode studies assessed patients at initial presentation during which they exhibited active psychotic symptoms that may obscure the potential relationships of SQoL with various symptom dimensions. It is also possible that predictors of SQoL may vary at different phases of illness (Bow-Thomas et al., 1999). Thus far, most prior FEP research primarily examined the impact of psychiatric symptoms on SQoL. There is a paucity of data in FEP regarding the relationship between SQoL and treatment-related variables including antipsychotic-induced extrapyramidal side-effects and attitude toward medications which were found to predict lower SQoL in chronic samples (Awad et al., 1997; Browne et al., 1998; Hofer et al., 2004). Although literature demonstrated that anxiety symptoms (Huppert and Smith, 2001; Braga et al., 2005), especially social anxiety (Pallanti et al., 2004; Kumazaki et al., 2012) which is the most prevalent comorbid anxiety condition in patients with psychotic disorders (Achim et al., 2011), were related to poorer SQoL in chronic schizophrenia, very few studies have been conducted to investigate the association between anxiety symptoms (and none for social anxiety) and SQoL in FEP. Further, most of these first-episode studies assessed anxiety symptoms by using either ratings of a single item (Theodore et al., 2012) or a symptom component score combining depression and anxiety items (Priebe et al., 2000; Melle et al., 2005; Gorna et al., 2008; MacBeth et al., 2015) selected from a general psychopathology scale. Such measurement lacks specificity to detect the influence of anxiety symptoms, in particular social anxiety, on SQoL.

Hong Kong (HK) is among the few cities in Asia to implement a territory-wide early intervention (EI) service for psychosis. The intervention program Early Assessment Service for Young People with Psychosis (EASY) was launched in 2001 and comprises three key elements including community-awareness programs, open referral system, and 2-year specialized intervention for young people presenting with FEP (Tang et al., 2010). We have previously examined the relationships of SQoL with clinical parameters in FEP patients at first presentation to EASY program, with depression being found to be the only variable correlating with SQoL (Law et al., 2005). In the current report, we sought to identify the determinants of SQoL in a representative cohort of Chinese FEP patients upon completion of 2-year treatment from EASY program. Comprehensive evaluation of depression, social anxiety, medication side-effects and attitude toward antipsychotic treatment, alongside standard measures of premorbid adjustment, psychopathology and functioning was conducted to enable a better delineation of prediction profiles for SQoL.

\section{Methods}

\subsection{Participants and setting}

Details of the study methodology have been reported elsewhere (Chang et al., 2015a). Briefly, the current analysis was based on data obtained from baseline assessment of a single-blind randomized controlled trial (RCT) comparing a 1-year extension of specialized EI (i.e., a 3-year EI service) with step-down care (i.e., a 2-year EI service) in patients who had received 2 years of treatment from EASY program. This publicly-funded specialized program provides early assessment and phase-specific intervention for all individuals aged 15-25 years experiencing their FEP in HK (Tang et al., 2010). The service consisted of five clinical teams, each covering a geographically-defined catchment area and comprising two psychiatrists, three case managers and one social worker. The service adopts phase-specific case-management approach in which each patient is assigned with a case manager who provides protocol-based psychosocial interventions (So, 2013). Patients are assertively followed up for a period of 2 years, after which they are man- aged by step-down clinic in the third year of treatment without provision of case-management. They are then transferred to generic psychiatric service for continuous care.

A total of 160 consecutive patients with a DSM-IV diagnosis of psychotic disorder were recruited from the outpatient units of EASY program between November 2010 and August 2011, randomly allocated to extended EI (Extended EI) or step-down care (SC), and followed up for 12 months. Exclusion criteria were intellectual disability, substance-induced psychosis or psychotic disorder due to general medical condition. In Extended EI condition, specialized EI was continued in the form of an additional year of case-management which was closely aligned with treatment protocols adopted by EASY program. Participants allocated to SC condition received outpatient medical follow-up with limited community support which focused mainly on crisis intervention, and no case-management was provided. Two treatment groups did not differ from each other regarding the intensity of medical follow-up by psychiatrists, prescription of antipsychotic medications, and availability of various psychosocial interventions and community-based services (Chang et al., 2015a).

In this report, we focused on investigating clinical, functional and treatment correlates of SQoL at study intake (i.e., upon completion of 2-year EASY program and prior to randomization) in 159 participants of the RCT cohort who were clinically stabilized with treatment and had completed SQoL assessment. The study was approved by the local institutional review boards. All participants provided written informed consent. For those aged under 18 years, consent was also obtained from a parent or guardian.

\subsection{Assessments}

\subsubsection{Diagnostic ascertainment}

Diagnosis of each participant was ascertained in consensus meetings attended by senior psychiatrist and research assistants using all available information including Chinese-bilingual Structured Clinical Interview for DSM-IV (CB-SCID, So et al., 2003), informant histories and medical records. Previous validation study showed that CB-SCID yielded reliable DSM-IV diagnoses in Chinese patients with psychotic disorders (So et al., 2003).

\subsubsection{Premorbid functioning and onset characteristics}

Premorbid functioning was measured with the Premorbid Adjustment Scale (PAS, Cannon-Spoor et al., 1982). We divided the PAS measure into academic and social premorbid functioning as adopted by previous reports (Larsen et al., 2004; Jeppesen et al., 2008; Chang et al., 2013) based on the results of prior factor-analytic studies on PAS which consistently demonstrated two separable domains (i.e., academic and social domains) in patients with psychotic disorders (Allen et al., 2001; MacBeth and Gumley, 2008). An overall score for each of the two premorbid functional domains was computed by averaging the ratings for the relevant subscales across developmental stages (score range $0-1$, higher score indicates lower functioning). Interview for the Retrospective Assessment of the Onset of Schizophrenia (IRAOS, Hafner et al., 1992) was employed to determine age at onset of psychosis and DUP which was defined as the time interval between the onset of positive psychotic symptoms and treatment initiation.

\subsubsection{Symptom severity and functioning}

Psychopathology was assessed using Positive and Negative Syndrome Scale (PANSS, Kay et al., 1987), Calgary Depression Scale (CAS, Addington et al., 1990), and Liebowitz Social Anxiety Scale (LSAS, Liebowitz, 1987). LSAS was shown to be a reliable instrument assessing social anxiety in schizophrenia patients (Pallanti et al., 2004). Psychosocial functioning was measured by Social and Occupational Functioning Assessment Scale (SOFAS, Goldman et al., 1992). 


\subsubsection{Medication side-effects and treatment attitude}

Antipsychotic-induced extrapyramidal side-effects including Parkinsonism features, akathisia and dyskinesia were assessed using Simpson-Angus Scale (SAS, Simpson and Angus, 1970), Barnes Akathisia Rating Scale (BARS, Barnes, 1989) and Abnormal Involuntary Movement Scale (AIMS, Guy, 1976), respectively. Patients' attitude toward antipsychotic medications were measured by a self-report 10-item Drug Attitude Inventory (DAI-10, Awad, 1993), with a higher score indicating more positive attitude.

\subsubsection{Subjective quality of life assessment}

Subjective health-related QoL was measured using Chinese version of Medical Outcome Study 36 Item Short Form Health Survey (SF-36, Ware and Sherbourne, 1992, Lam et al., 1998) which is a self-rated questionnaire assessing 8 domains including physical functioning, role limitation due to physical functioning, bodily pain, general health, vitality, social functioning, role limitation due to emotional problems, and mental health. Score of each domain ranges from 0 to 100, with higher score indicating better SQoL. SF-36 has been extensively used in clinical populations of physical and mental illnesses including schizophrenia (Nasrallah et al., 2004; Leese et al., 2008). Literature has shown that SF-36 is a reliable and valid measure of health-related SQoL for patients with psychotic disorders (Tunis et al., 1999; Meijer et al., 2002). Chinese version of SF-36 was validated in healthy participants in a population-based study (Lam et al., 1998) and has been studied in Chinese FEP sample in HK (Law et al., 2005). In this study, mental and physical component summary (MCS and PCS) scores (McHorney et al., 1993; Lam et al., 1998; Leese et al., 2008) were computed as primary measures of SQoL.

Research assistants who received systematic training on the use of study instruments before participant recruitment and masked to treatment allocation administered all assessments. Videotaped interviews of 10 cases were selected and independently rated by research assistants for the purpose of inter-rater reliability evaluation. Intra-class correlation coefficients (ICCs) for PANSS general psychopathology, positive and negative symptom subscales, CDS total score and SOFAS score were $0.92,0.96,0.91,0.89$ and 0.93 , respectively, indicating good inter-rater reliability (Chang et al., 2015a).

\subsection{Statistical analysis}

The primary analysis focused on identifying predictors of SF-36 MCS and PCS scores. First, associations of SF-36 MCS and PCS scores with demographics, premorbid adjustment, and clinical, functional and treatment-related variables were examined using Pearson's product-mean correlation analysis (continuous candidate variables) and independent t-test (dichotomous candidate variables) as appropriate. Then, those variables that were found to be significantly related to SF-36 MCS and PCS scores in the preceding analyses were entered into multiple linear regression models to determine which factors independently predicted these two SF-36 QoL measures. All analyses were performed with the SPSS, version 24.0, and the level of statistical significance was set at $\mathrm{p}<0.05$.

\section{Results}

\subsection{Characteristics of the sample}

Demographics, premorbid adjustment, clinical and treatment-related characteristics, and functioning of the study sample are summarized in Table 1. Of 159 participants, 50.9\% were male. The mean age of the sample was 22.9 years (S.D. $=3.19$ ) and the median DUP was 13 weeks $($ mean $=36$, S.D. $=52.5)$. Diagnoses of the cohort were schizo-
Table 1

Demographics, premorbid adjustment, clinical and treatment characteristics of the study sample.

\begin{tabular}{ll}
\hline Variables & Mean (S.D.) / N (\%) \\
\hline Demographics & \\
Age at entry & $22.87(3.19)$ \\
Male gender & $81(50.94)$ \\
Tertiary education level or above & $41(25.79)$ \\
Premorbid adjustment & \\
PAS social domain score & \\
PAS academic domain score & $0.43(0.43)$ \\
Illness characteristics and allocated treatment & $0.41(0.39)$ \\
Age at onset of psychosis & \\
DUPb, weeks & $20.21(3.13)$ \\
Psychiatric diagnosis & $36(52.50)$ \\
Schizophrenia-spectrum disorder & \\
Affective psychosis & \\
Other non-affective psychoses & $131(82.39)$ \\
Symptom severity and functioning & $15(9.43)$ \\
PANSS positive symptom score & $13(8.18)$ \\
PANSS negative symptom score & \\
CDS total score & $9.21(3.07)$ \\
LSAS social anxiety total score & $11.91(5.24)$ \\
SOFAS score & $2.87(3.44)$ \\
Antipsychotics \& Medication side-effect & $37.16(33.50)$ \\
Use of SGA & $57.67(13.98)$ \\
CPZ equivalents & \\
SAS score & $132(83.0)$ \\
BARS score & $303.46(253.66)$ \\
AIMS score & $2.80(3.53)$ \\
Subjective quality of life & $1.59(2.55)$ \\
SF36 mental component summary score & $1.54(2.52)$ \\
SF36 physical component summary score & $42.93(11.84)$ \\
\hline & $48.66(8.94)$ \\
\hline
\end{tabular}

Abbreviations: AIMS = Abnormal Involuntary Movement Scale; BARS = Barnes Akathisia Rating Scale; CDS = Calgary Depression Scale; $\mathrm{CPZ}=$ Chlorpromazine; DUP $=$ Duration of untreated psychosis; LSAS = Liebowitz Social Anxiety Scale; PANSS = Positive and Negative Syndrome Scale; PAS = Premorbid adjustment scale; SAS = Simpson-Angus Scale; SF-36= Medical Outcome study 36 Item Short Form Health Survey; SGA, Second-generation antipsychotic; SOFAS $=$ Social and Occupational Functioning Assessment Scale.

a PAS social domain comprised 3 of the 5 PAS subscales including sociability and withdrawal, peer relationships, and social-sexual functioning. PAS academic domain comprised 2 of the 5 PAS subscales including scholastic performance and adaptation to school (Allen et al., 2001; Chang et al., 2013).

b Median DUP was 13 weeks.

c Schizophrenia-spectrum disorder included schizophrenia $(n=98)$, schizoaffective disorder $(n=5)$ and schizophreniform disorder $(n=28)$.

d Affective psychosis included bipolar affective disorder with psychotic symptoms $(\mathrm{n}=14)$ and depressive disorder with psychotic symptoms $(\mathrm{n}=1)$.

e Other non-affective psychoses included brief psychotic disorder $(n=3)$, delusional disorder $(n=9)$ and psychosis not otherwise specified $(n=1)$.

phrenia-spectrum disorder $(n=131)$, affective psychosis $(n=15)$ and other non-affective psychoses $(n=14)$.

\subsection{Predictors of SF-36 QoL measures in multivariate models}

As shown in Table 2, both SF-36 MCS and PCS scores were significantly associated with ratings on key symptom dimensions including PANSS positive and negative symptom scores, depression (CDS total score) and social anxiety levels (LSAS total score), as well as psychosocial functioning (SOFAS score). SF-36 MCS score was also found to be significantly related to PAS social domain score, age at onset of psychosis, antipsychotic-induced extrapyramidal side-effects including Parkinsonism features (SAS score) and akathisia (BARS score), and attitude toward antipsychotic medications (DAI-10 score). There were no significant relationships of SF-36 PCS score with demographics, premorbid adjustment, illness characteristics, and treatment and side-effect variables.

Multiple regression analysis revealed that positive symptom severity, depression and social anxiety levels, attitude toward antipsychotic medications, and degree of akathisia independently predicted SF-36 MCS score, accounting for $47.8 \%$ of the variance (Table 3). SF-36 PCS 
Table 2

Relationships of SF36 QoL measures with demographics, premorbid adjustment, clinical and treatment characteristics. ${ }^{\mathrm{a}}$

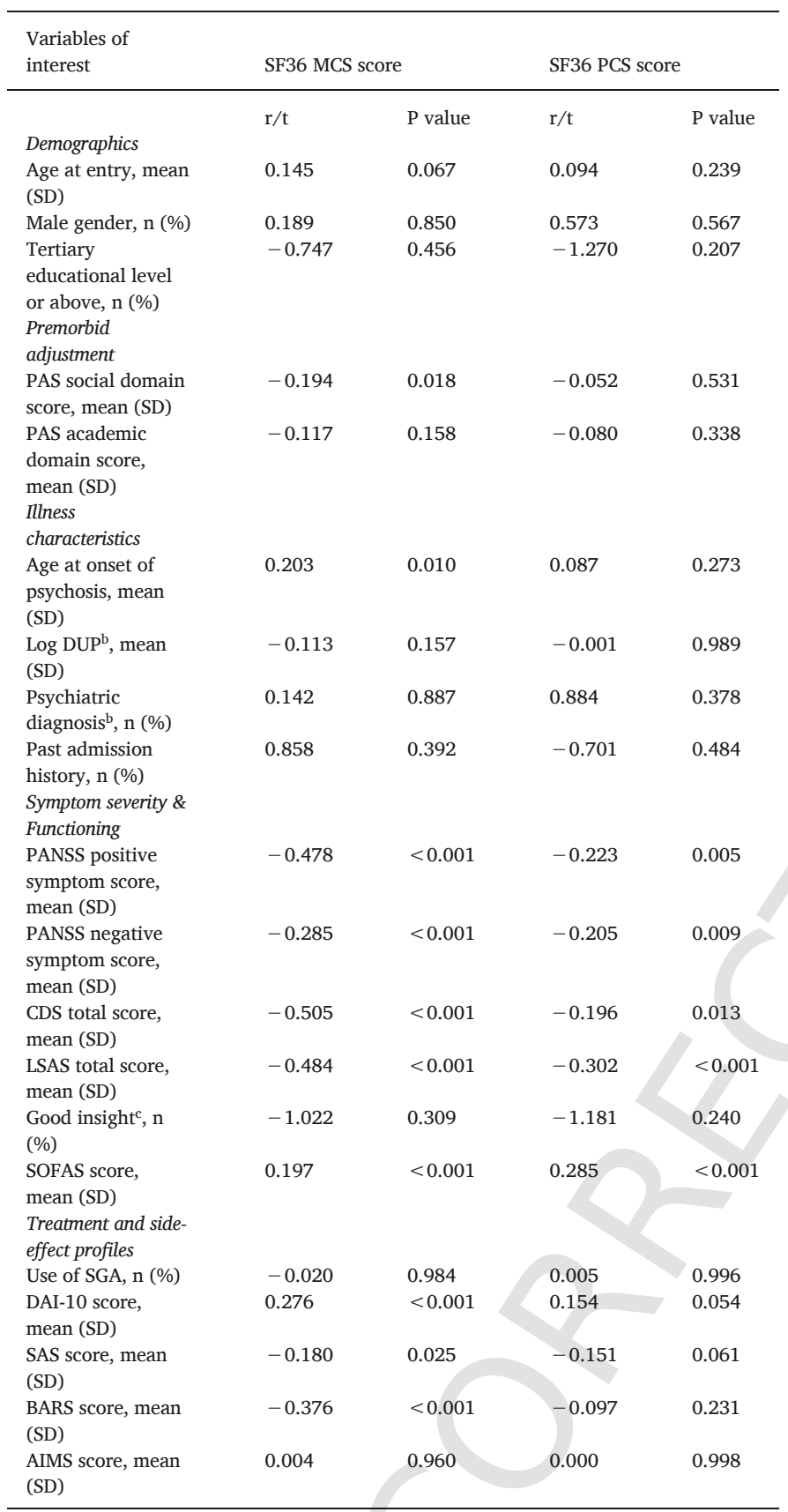

Abbreviations: AIMS = Abnormal Involuntary Movement Scale; BARS = Barnes Akathisia Rating Scale; $\mathrm{CDS}=$ Calgary Depression Scale; DAI = Drug Attitude Inventory; DUP = Duration of untreated psychosis; LSAS = Liebowitz Social Anxiety Scale; MCS = Mental component summary; PANSS = Positive and Negative Syndrome Scale; PAS = Premorbid adjustment scale; PCS = Physical component summary; QoL = Quality of life; SAS = Simpson-Angus Scale; SGA = Second-generation antipsychotic; SF-36 = Medical Outcome study 36 Item Short Form Health Survey; SOFAS = Social and Occupational Functioning Assessment Scale.

a Pearson's product-mean correlation analyses and independent t-tests were performed to examine the relationships of SF36 scores with continuous and categorical variables, respectively.

b DUP was log-transformed due to its skewed distribution.

c Psychiatric diagnosis was categorized into schizophrenia-spectrum disorder (included schizophrenia, schizoaffective or schizopreniform disorder) and other psychotic disorders (included bipolar affective disorder with psychotic symptoms, depressive disorder with psychotic features, brief psychotic disorder, delusional disorder and psychosis not otherwise specified) for analysis. Good insight was defined as PANSS item G12 rating $<4$.
Table 3

Final multiple regression model for prediction of SF36 mental component summary score. $^{\mathrm{a}, \mathrm{b}}$.

\begin{tabular}{|c|c|c|c|c|}
\hline $\begin{array}{l}\text { Variables in the } \\
\text { equation }\end{array}$ & B & $\mathrm{t}$ & $P$ value & $95 \% \mathrm{CI}$ \\
\hline $\begin{array}{l}\text { PANSS positive } \\
\text { symptom score }\end{array}$ & -1.076 & -4.007 & $<0.001$ & $\begin{array}{l}-1.608 \\
\text { to }\end{array}$ \\
\hline CDS total score & -0.844 & -3.444 & 0.001 & $\begin{array}{l}-1.328 \\
\text { to } \\
-0.359\end{array}$ \\
\hline LSAS total score & -0.122 & -3.963 & $<0.001$ & $\begin{array}{l}-0.183 \\
\text { to } \\
-0.061\end{array}$ \\
\hline DAI-10 score & 0.455 & 2.591 & 0.011 & $\begin{array}{l}0.108- \\
0.803\end{array}$ \\
\hline BARS score & -0.954 & -0.204 & 0.002 & $\begin{array}{l}-1.547 \\
\text { to } \\
-0.361\end{array}$ \\
\hline Constant & 60.175 & 24.169 & $<0.001$ & $\begin{array}{l}55.251 \\
- \\
65.099\end{array}$ \\
\hline
\end{tabular}

Abbreviations: BARS = Barnes Akathisia Rating Scale; CDS = Calgary Depression Scale; DAI = Drug Attitude Inventory; LSAS = Liebowitz Social Anxiety Scale; PANSS = Positive and Negative Syndrome Scale;PAS = Premorbid adjustment scale; SAS = Simpson-Angus Scale; SF36 = Medical Outcome study 36 Item Short Form Health Survey; SOFAS = Social and Occupational Functioning Assessment Scale.

a PAS social domain score, age at onset of psychosis, PANSS negative symptom score, SOFAS score and SAS score, which were entered into the multiple regression model, were excluded as predictors of SF36 mental health score after analysis.

b Final Model: adjusted $\mathrm{R}^{2}=0.478, \mathrm{~F}=26.438, \mathrm{p}<0.001$.

score was predicted by social anxiety and functional levels which accounted for $12.2 \%$ of the variance (Table 4 ).

\section{Discussion}

The aim of this study was to investigate clinical and treatment-related determinants of SQoL in Chinese FEP patients who had received 2-year specialized EI service. Consistent with most previous first-episode studies (Malla et al., 2004; Sim et al., 2004; Melle et al., 2005; Wegener et al., 2005; Cotton et al., 2010; Renwick et al., 2012; Gardsjord et al., 2016), the current report showed that depressive symptoms independently predicted mental SQoL, thereby further confirming a critical role of depression in determining subjective mental wellbeing in FEP patients. This finding also extend our prior work on examining clinical correlates of SQoL in FEP patients at service intake (Law et al., 2005), and indicates depression as a robust predictor of SQoL in both acute and recovery phase of FEP. The current study, to

Table 4

Final multiple regression model for prediction of SF36 physical component summary score. ${ }^{\mathrm{a}, \mathrm{b}}$.

\begin{tabular}{lllll}
\hline $\begin{array}{l}\text { Variables in the } \\
\text { equation }\end{array}$ & $\mathrm{B}$ & $\mathrm{t}$ & P value & $95 \% \mathrm{CI}$ \\
\hline LSAS total score & -0.091 & -3.385 & 0.001 & $\begin{array}{l}-0.145 \\
\text { to } \\
-0.038\end{array}$ \\
SOFAS score & 0.138 & 2.713 & 0.007 & $0.038-$ \\
& 60.175 & 24.169 & $<0.001$ & $\begin{array}{l}5.239 \\
-\end{array}$ \\
Constant & & & & 65.099 \\
& & & & \\
\end{tabular}

Abbreviations: CDS = Calgary Depression Scale; LSAS = Liebowitz Social Anxiety Scale; PANSS = Positive and Negative Syndrome Scale; SF36 = Medical Outcome study 36 Item Short Form Health Survey; SOFAS = Social and Occupational Functioning Assessment Scale.

a PANSS positive symptom score, PANSS negative symptom score, and CDS total score, which were entered into the multiple regression model, were excluded as predictors of SF36 physical health score after analysis.

b Final Model: adjusted $\mathrm{R}^{2}=0.122, \mathrm{~F}=11.551, \mathrm{p}<0.001$. 
our knowledge, is the first to examine the influence of social anxiety on SQoL in FEP patients. The results revealed that social anxiety significantly contributed to mental and physical SQoL and thus concur with the literature on chronic schizophrenia showing that patients with comorbid social anxiety disorder had lower SQoL than those without social anxiety disorder (Pallanti et al., 2004). One recent study further demonstrated that worsening of social anxiety symptoms over 5 years was associated with decreased SQoL in schizophrenia patients (Kumazaki et al., 2012). In fact, this is in line with previous reviews regarding the influence of anxiety disorders on SQoL (Mendlowicz and Stein, 2000; Olatunji et al., 2007) which found that social anxiety disorder or even subthreshold social anxiety symptoms negatively impacted on an individual's functioning independent of sociodemographic and other clinical variables, resulting in an increased dissatisfaction with life. Owing to the scarcity of existing data, further research is required to confirm our findings of unique contribution of social anxiety to SQoL in FEP patients.

Contrary to many studies (Malla et al., 2004; Melle et al., 2005; Wegener et al., 2005; Renwick et al., 2012; MacBeth et al., 2015) which suggested a lack of association between positive symptoms and SQoL in FEP samples, our result showed that positive symptoms negatively influenced mental SQoL. Nonetheless, this finding is consistent with several first-episode studies (Chee et al., 2010; Cotton et al., 2010; Thorup et al., 2010) which examined SQoL at clinical stabilization instead of at initial presentation and revealed that positive symptoms predicted SQoL. In fact, this is in agreement with previous research which indicated that the association between positive symptoms and SQoL was stronger when patients were at clinical stabilization than during the acute phase of psychosis (Bow-Thomas et al., 1999). This finding also concurs with past studies on Chinese schizophrenia outpatients which found that positive symptom severity was inversely related to SQoL (Xiang et al., 2007, 2008). It is postulated that the traditional Chinese cultural values that emphasize modesty and oppose extremes may lead to highly negative attitudes of the general public toward positive psychotic symptoms, which are perceived as "unpredictable" and "potential threats to safety" (Xiang et al., 2010). The stigmatizing attitudes and discrimination experienced by patients with psychotic disorders may negatively impact on their self-esteem and SQoL. Our result that functioning predicted physical SQoL is in keeping with results of earlier studies which showed that impaired global functioning was associated with various aspects of perceived life satisfaction including physical domain in FEP patients (Cotton et al., 2010; Melle et al., 2010; Renwick et al., 2015).

The influence of antipsychotic-induced side-effects and patients' attitude toward medication has, thus far, not been adequately investigated in FEP. Yet, FEP patients perceived negative impact of antipsychotic treatment as one of the major barriers toward recovery (Lam et al., 2011). Consistent with results of previous studies on chronic schizophrenia (Awad et al., 1997; Browne et al., 1998; Hofer et al., 2004), we found that antipsychotic-induced akathisia and negative attitude toward antipsychotic treatment were associated with poorer mental SQoL. This echoes findings of our prior research on adult-onset FEP which demonstrated differential relationships of various antipsychotics with SQoL, with those medications having higher propensity to induce extrapyramidal side-effects being related to lower SQoL (Lee et al., 2015). In fact, akathisia, which is characterized by motor and subjective inner restlessness, has been regarded as one of the most distressing antipsychotic-induced movement disorders (Poyurovsky, 2010). It is also acknowledged that akathisia is a risk factor for suicidal behavior, particularly when it is coupled with depression and impulsivity. Our finding regarding the effect of negative treatment attitude is in line with one recent first-episode study which revealed that patients' beliefs about treatment control predicted SQoL (Theodore et al., 2012).
The findings of a lack of significant association between negative symptoms and SQoL are contrary to some (Malla et al., 2004; Gorna et al., 2008; Cotton et al., 2010; Thorup et al., 2010), but not all previous studies which revealed that higher levels of negative symptoms adversely impacted on SQoL in FEP patients. One possible explanation for such discrepancy is the variation in negative symptom measurement across studies. Most of those studies (Malla et al., 2004; Cotton et al., 2010; Thorup et al., 2010) that found significant associations employed a Scale for Assessment of Negative Symptoms (SANS, Andreasen, 1982) which is specifically designed to assess individual sub-domains of negative symptom construct and thus provides a more refined and sensitive negative symptom measurement when compared to PANSS (Blanchard and Cohen, 2006) (as adopted in the current study), enabling the investigators to detect subtle associations with SQoL. It might also be possible that the use of two aggregate SQoL scores instead of individual SF36 domain scores might mask the potential significant relationships of these SQoL domains with negative symptoms. Alternatively, mixed findings were noted regarding the relationship between DUP and SQoL. Some studies showed that DUP was negatively correlated with SQoL (Malla et al., 2004; Melle et al., 2005; Gorna et al., 2008). However, consistent with other previous research (Ho et al., 2000; Law et al., 2005; Thorup et al., 2010; Cotton et al., 2010; MacBeth et al., 2015), we found no significant association between DUP and SQoL in our FEP sample. The null finding might, on the other hand, be attributed to the comparatively short DUP (median DUP: 13 weeks) of our cohort which might then obscure the potential negative impact of prolonged untreated psychosis on SQoL.

Results of the current study have several clinical implications with regard to SQoL enhancement. First, given that depression and social anxiety are major determinants of SQOL in FEP patients and are highly prevalent in the early stage of illness (Voges and Addington, 2005; Cotton et al., 2012; Chang et al., 2015b), regular and systematic evaluation of these affective symptoms should be integrated in EI service to facilitate early detection and intervention which will in turn promote patients' SQoL. Second, treatment for positive symptoms should be optimized to minimize the risk of emergence of residual symptoms or psychosis exacerbation which negatively influences SQoL. Third, treatment-related variables also play a crucial role in determining SQoL and represent modifiable therapeutic targets in FEP. Thus, close monitoring and prompt management of antipsychotic-induced side-effects should be provided. Comprehensive psychoeducation on antipsychotic treatment should be offered to patients, particularly in the initial treatment phase, to properly address their misconception and negative attitude toward medications. Additionally, more intensive psychosocial interventions should be incorporated to EI service to promote early functional recovery which is linked to better SQoL.

There are several limitations in the study. First, we did not include measures on service satisfaction, illness coping styles and cognitive functioning which have been suggested as potential predictors of SQoL in schizophrenia (Ritsner et al., 2003; Wegener et al., 2005; Petkari and Pietschnig, 2015). Second, SF-36 is a generic SQoL instrument which may not adequately capture SQoL domains that are specifically relevant to patients with psychotic disorders. Third, the study sample was recruited from EASY program which provides EI to patients aged 15-25 years. Hence, our results may not be generalizable to those with older age of psychosis onset. Fourth, the current analysis focused on FEP patients at clinical stabilization who exhibited relatively less severe positive symptoms. This limits the comparability of our findings with those first-episode studies which examined patients upon their initial presentation with active psychosis. Fifth, although literature has supported the validity and reliability of self-report measures of patients with psychotic disorders, the subjective nature of SQoL evaluation may still be biased by patients' degrees of symptom severity, insight and cognitive impairment, as well as their willingness to cooperate with the 
assessment. Sixth, the cross-sectional design precluded us from drawing conclusions about causal relationship between identified correlates and SQoL measures. Longitudinal research is required to clarify the course and prospective predictors of SQoL in the early course of psychotic illness.

\section{Conflicts of interest}

Author E.Y.H.C. has participated in the paid advisory board for Otsuka, has received educational grant support from Janssen-Cilag, and has received research funding from Astra-Zeneca, Janssen-Cilag, Eli Lilly, Sanofi-Aventis and Otsuka. The authors report no conflicts of interest. The authors alone are responsible for the content and writing of the paper.

\section{Acknowledgement}

This study was supported by a grant from the Commissioned Research on Mental Health Policy and Services (SMH-29) of the Food and Health Bureau, the Government of Hong Kong Special Administrative Region.

\section{References}

Achim, A.M., Maziade, M., Raymond, E., Oiver, D., Merette, C., Roy, M.A., 2011. How prevalent are anxiety disorders in schizophrenia? A meta-analysis and critical review on a significant association. Schizophr. Bull. 37, 811-821.

Addington, D., Addington, J., Schissel, B., 1990. A depression rating scale for schizophrenics. Schizophr. Res. 3, 247-251.

Allen, D.N., Kelley, M.E., Miyatake, R.K., Gurklis, J.A., van Kammen, D.P., 2001. Confirmation of a two-factor model of premorbid adjustment in males with schizophrenia. Schizophr. Bull. 27, 39-46.

Andreasen, N.C., 1982. Negative symptoms in schizophrenia. Definitions and reliability. Arch. Gen. Psychiatry 39, 784-788.

Awad, A.G., 1993. Subjective response to neurolcptics in schirophrenia. Schizophr. Bull. 19, 609-618.

Awad, A.G., Voruganti, L.N.P., Heslegrave, R.J., 1997. A conceptual model of quality of life in schizophrenia: a description and preliminary clinical validation. Qual. Life Res. 6, 21-26.

Barnes, T.R., 1989. A rating scale for drug-induced Akathisia. Br. J. Psychiatry $154,672-676$.

Blanchard, J.J., Cohen, A.S., 2006. The structure of negative symptoms within schizophrenia: implications for assessment. Schizophr. Bull. 32, 238-245.

Bow-Thomas, C.C.1, Velligan, D.I., Miller, A.L., Olsen, J., 1999. Predicting quality of life from symptomatology in schizophrenia at exacerbation and stabilization. Psychiatry Res. 86, 131-142.

Boyer, L., Millier, A., Perthame, E., Abellea, S., Auquier, P., Toumi, M., 2013. Quality of life is predictive of relapse in schizophrenia. BMC Psychiatry 13, 15.

Boyer, L., Lancon, C., Karine, B., Parola, N., Berbis, J., Acquier, P., 2013. Evaluating the impact of a quality of life assessment with feedback to clinicians in patients with schizophrenia: randomized controlled trial. Br. J. Psychiatry 202, 447-453.

Braga, R.J., Mendlowicz, M.V., Marrocos, R.P., Figueira, I.L., 2005. Anxiety disorders in outpatients with schizophrenia: prevalence and impact on the subjective quality of life. J. Psychiatr. Res. 39, 409-414.

Browne, S., Garavan, J., Gervin, M., et al., 1998. Quality of life in schizophrenia: insight and subjective response to neuroleptics. J. Nerv. Ment. Dis. 186, 74-78.

Cannon-Spoor, H.E., Potkin, S.G., Wyatt, R.J., 1982. Measurement of premorbid adjustment in chronic schizophrenia. Schizophr. Bull. 8, 470-484.

Chang, W.C., Tang, J.Y.M., Hui, C.L.M., et al., 2013. The relationship of early premorbid adjustment with negative symptoms and cognitive functions in first-episode schizophrenia: a prospective three-year follow-up study. Psychiatry Res. 209, 353-360.

Chang, W.C., Chan, G.H.K., Jim, O.T.T., et al., 2015. Optimal duration of an early intervention programme for first-episode psychosis: randomised controlled trial. Br. J. Psychiatry 206, 492-500.

Chang, W.C., Cheung, R., Hui, C.L.M., et al., 2015. Rate and risk factors of depressive symptoms in Chinese patients presenting with first-episode non-affective psychosis in Hong Kong. Schizophr. Res. 168, 99-105.

Chee, K.Y., 2010. Determinants of subjective quality of life in first-episode schizophrenia: perspective from Malaysia. Early Interv. Psychiatry 4, 111-118.

Cotton, S.M., Gleeson, J., Alvarez-Jimenez, M., McGorry, P.D., 2010. Quality of life in patients who have remitted from their first episode of psychosis. Schizophr. Res. 121, 259-265.

Cotton, S.M., Lambert, M., Schimmelmann, B.G., Mackinnon, A., Gleeson, J.F., Berk, M., Hides, L., Chanen, A., McGorry, P.D., Conus, P., 2012. Depressive symptoms in first episode schizophrenia spectrum disorder. Schizophr. Res. 134, 20-26.

Eack, S.M., Newhill, C.E., 2007. Psychiatric symptoms and quality of life in schizophrenia: a meta-analysis. Schizophr. Bull. 33, 1225-1237.

Gardsjord, E.S., Romm, K.L., Friis, S., Barder, H.E., Evensen, J., Haahr, U., Ten Velden Hegelstad., W., Joa, I., Johannessen, J.O., Langeveld, J., Larsen, T.K., Opjordsmoen, S., Rund, B.R., Simonsen, E., Vaglum, P., McGlashan, T., Melle, I., Røssberg, J.I.,
2016. Subjective quality of life in first-episode psychosis. A ten year follow-up study. Schizophr. Res. 172, 23-28.

Goldman, H.H., Skodol, A.E., Lave, T.R., 1992. Revising Axis-V for DSM-IV: a review of measures of social functioning. Am. J. Psychiatry 149, 1148-1156.

Górna, K., Jaracz, K., Rybakowski, F., Rybakowski, J., 2008. Determinants of objective and subjective quality of life in first-time-admission schizophrenic patients in Poland: a longitudinal study. Qual. Life Res. 17, 237-247.

Guy, W. 1976. Abnormal Involuntary Movement Scale (AIMS). ECDEU Assessment Manual for Psychopharmacology, Revised, pp. 534-537.

Häfner, H., Riecher-Rössler, A., Hambrecht, M., Maurer, K., Meissner, S., Schmidtke, A., Fätkenheuer, B., Löffler, W., van der Heiden, W., 1992. IRAOS: an instrument for the assessment of onset and early course of schizophrenia. Schizophr. Res. 6, 209-223.

Ho, B.C., Andreasen, N.C., Flaum, M., Nopoulos, P., Miller, D., 2000. Untreated initial psychosis: its relation to quality of life and symptom remission in first-episode schizophrenia. Am. J. Psychiatry 157, 808-815.

Hofer, A., Gemmler, G., Eder, U., Edlinger, M., Hummer, M., Fleischhacker, W.W., 2004 Quality of life in schizophrenia: the impact of psychopathology, attitude toward medication, and side effects. J. Clin. Psychiatry 65, 932-939.

Huppert, J.D., Smith, T.E., 2001. Longitudinal analysis of subjective quality of life in schizophrenia: anxiety as the best symptom predictor. J. Nerv. Ment. Dis. 189, 669-675.

Jeppesen, P., Petersen, L., Thorup, A., Abel, M.B., Ohlenschlaeger, J., Christensen, T.O., Krarup, G., Jorgensen, P., Nordentoft, M., 2008. The association between pre-morbid adjustment, duration of untreated psychosis and outcome in first-episode psychosis. Psychol. Med. 38, 1157-1166.

Karow, A., Wittmann, L., Schottle, D., Schafer, I., Lambert, M., 2014. The assessment of quality of life in clinical practice in patients with schizophrenia. Dialogues Clin. Neurosci. 16, 185-195.

Kay, S.R., Flszbein, A., Opfer, L.A., 1987. The Positive and Negative Syndrome Scale (PANSS) for schizophrenia. Schizophr. Bull. 13, 261-276.

Kumazaki, H., Kobayashi, H., Niimura, H., Kobayashi, Y., Ito, S., Nemoto, T., Sakuma, K., Kashima, H., Mizuno, M., 2012. Lower subjective quality of life and the development of social anxiety symptoms after the discharge of elderly patients with remitted schizophrenia: a 5-year longitudinal study. Compr. Psychiatry 53, 946-951.

Lam, C.L.K., Gandek, B., Ren, X.S., 1998. Tests of scaling assumptions and construct validity of the Chinese (HK) version of the SF-36 Health Survey. J. Clin. Epidemiol. 51, 1139-1147.

Lam, M.M.L., Pearson, V., Ng, R.M.K., Chiu, C.P.Y., Law, C.W., Chen, E.Y.H., 2011. What dose recovery from psychosis mean? Perceptions of young first-episode patients. Int. J. Soc. Psychiatry 57, 580-587.

Lambert, M., Naber, D., Eich, F.X., Schacht, M., Linden, M., Schimmelmann, B.G., 2007. Remission of severely impaired subjective wellbeing in 727 patients with schizophrenia treated with amisulpride. Acta Psychiatr. Scand. 115, 106-113.

Larsen, T.K., Friis, S., Haahr, U., Johannessen, J.O., Melle, I., Opjordsmoen, S., Rund, B.R., Simonsen, E., Vaglum, P.V., McGlashan, T.H., 2004. Premorbid adjustment in first-episode non-affective psychosis: distinct patterns of pre-onset course. Br. J. Psychiatry $185,108-115$

Law, C., Chen, E.Y., Cheung, E.F., Chan, R.C., Wong, J.G., Lam, C.L., Leung, K.F., Lo, M.S., 2005. Impact of untreated psychosis on quality of life in patients with first-episode schizophrenia. Qual. Life Res. 14, 1803-1811.

Lee, E.H.M., Hui, C.L.M., Lin, J.J., Ching, E.Y., Chang, W.C., Chan, S.K., Chen, E.Y., 2015 Quality of life and functioning in first-episode psychosis Chinese patients with different antipsychotic medications. Early Interv. Psychiatry http://dx.doi.org/10.1111/ eip.12246.

Leese, M., Schene, A., Koeter, M., et al., 2008. SF-36 scales, and simple sums of scales, were reliable quality-of-life summaries for patients with schizophrenia. J. Clin. Epidemiol. 616, 588-596.

Lehman, A.F., 1996. Measures of quality of life among persons with severe and persistent mental disorders. Soc. Psychiatry Psychiatr. Epidemiol. 31, 78-88.

Liebowitz, M.R., 1987. Social Phobia. Karger Publishers.

MacBeth, A., Gumley, A., 2008. Premorbid adjustment, symptom development and quality of life in first episode psychosis: a systematic review and critical appraisal. Acta Psychiatr. Scand. 117, 85-99.

MacBeth, A., Gumley, A., Schwannauer, M., Fisher, R., 2015. Self-reported quality of life in a Scottish first-episode psychosis cohort: associations with symptomatology and premorbid adjustment. Early Interv. Psychiatry 9, 53-60.

Malla A. Payne J. 2005. First-episode psychosis: psychopathology, quality of life, and functional outcome Schizophr. Bull. 31 650-671

Malla A. Norman R. McLean T. MacDonald C. McIntosh E. Dean-Lashley F. Lynch J. Scholten D. Ahmed R. 2004. Determinants of quality of life in first-episode psychosis Acta Psychiatr. Scand. 109 46-54

McHorney, C.A., Ware, J.E., Raczek, A.E., 1993. The MOS SF-36 Item Short-Form Health Survey (SF-36) II: psychometric and clinical tests of validity in measuring physical and mental health constructs. Med. Care 31, 247-263.

Meijer, C.J., Schene, A.H., Koeter, M.W.J., 2002. Quality of life in schizophrenia measured by the MOS SF-36 and the Lancashire Quality of Life Profile: a comparison. Acta Psychiatr. Scand. 105, 293-300.

Melle, I., Friis, S., Haahr, U., Johannesen, J.O., Larsen, T.K., Opjordsmoen, S., Roessberg, J.I., Rund, B.R., Simonsen, E., Vaglum, P., McGlashan, T., 2005. Measuring quality of life in first-episode psychosis. Eur. Psychiatry 20, 474-483.

Melle, I., Røssberg, J.I., Joa, I., Friis, S., Haahr, U., Johannessen, J.O., Larsen, T.K., Opjordsmoen, S., Rund, B.R., Simonsen, E., Vaglum, P., McGlashan, T., 2010. The development of subjective quality of life over the first 2 years in first-episode psychosis. J. Nerv. Ment. Dis. 198, 864-869.

Mendlowicz, M.V., Stein, M.B., 2000. Quality of life in individuals with anxiety disorders. Am. J. Psychiatry 157, 669-682.

Nasrallah, H.A., Duchesne, I., Mehnert, A., Janagap, C., Eerdekens, M., 2004. Health-related quality of life in patients with schizophrenia during treatment with long-acting, injectable risperidone. J. Clin. Psychiatry 65, 531-536. 
Olatunji, B.O., Cisler, J.M., Tolin, D.F., 2007. Quality of life in the anxiety disorders: a meta-analytic review. Clin. Psychol. Rev. 27, 572-581.

Pallanti, S., Quercioli, L., Hollander, E., 2004. Social anxiety in outpatients with schizophrenia: a relevant cause of disability. Am. J. Psychiatry 161, 53-58.

Petkari, E., Pietschnig, J., 2015. Associations of quality of life with service satisfaction in psychotic patients: a meta-analysis. PLoS One 10, e0135267.

Poyurovsky, M., 2010. Acute antipsychotic-induced akathisia revisited. Br. J. Psychiatry 196, 89-91.

Priebe, S., Roeder-Wanner, U.-U., Kaiser, W., 2000. Quality of life in first-admitted schizophrenia patients: a follow-up study. Psychol. Med. 30, 225-230.

Renwick, L., Jackson, D., Foley, S., Owens, E., Ramperti, N., Behan, C., Anwar, M., Kinsella, A., Turner, N., Clarke, M., O'Callaghan, E., 2012. Depression and quality of life in first-episode psychosis. Compr. Psychiatry 53, 451-455.

Renwick, L., Drennan, J., Sheridan, A., Owens, L., Lyne, J., O'Donoghue, B., Kinsella, A., Turner, N., O'Callaghan, E., Clarke, M., 2015. Subjective and objective quality of life at first presentation with psychosis. Early Interv. Psychiatry http://dx.doi.org/10.1111/ eip.12255.

Ritsner, M., Ben-Avi, I., Ponizovasky, A., Timinsky, I., Bistrov, E., Modai, I., 2003. Quality of life and coping with schizophrenia symptoms. Qual. Life Res. 12, 1-9.

Sim, K., Mahendran, R., Siris, S.G., Heckers, S., Chong, S.A., 2004. Subjective quality of life in first episode schizophrenia spectrum disorders with comorbid depression. Psychiatry Res. 129, 141-147.

Simpson, G.M., Angus, J.W., 1970. A rating scale for extrapyramidal side effects. Acta Psychiatr. Scand. 212 (suppl), 11-19.

So, E., Kam, I., Leung, C., Chung, D., Liu, Z., Fong, S., 2003. The Chinese-bilingual SCID-I/ P project: stage 1-reliability for mood disorders and schizophrenia. H. K. J. Psychiatry $13,7-18$.

So, S., 2013. Implementing Psychological Intervention Programme in Early Psychosis (PIPE). Early Psychos. Interv.: A Cult. Adapt. Clin. Guide 137-157.

Tang, J.Y., Wong, G.H., Hui, C.L., Lam, M.M., Chiu, C.P., Chan, S.K., Chung, D.W., Tso, S., Chan, K.P., Yip, K.C., Hung, S.F., Chen, E.Y., 2010. Early intervention for psychosis in Hong Kong--the EASY programme. Early Interv. Psychiatry 4, 214-219.
Theodore, K., Johnson, S., Chalmers-Brown, A., Doherty, R., Harrop, C., Ellett, L., 2012. Quality of life and illness beliefs in individuals with early psychosis. Soc. Psychiatry Psychiatr. Epidemiol. 47, 545-551.

Thorup, A., Petersen, L., Jeppesen, P., Nordentoft, M., 2010. The quality of life among first-episode psychotic patients in the opus trial. Schizophr. Res. 116, 27-34.

Tunis, S.L., Croghan, T.W., Heilman, D.K., Johnstone, B.M., Obenchain, R.L., 1999. Reliability, validity, and application of the Medical Outcomes Study 36-Item Short-Form Health Survey (SF-36) in schizophrenic patients treated with olanzapine versus haloperidol. Med. Care 37, 678-691.

Voges, M., Addington, J., 2005. The association between social anxiety and social functioning in first episode of psychosis. Schizophr. Res. 76, 287-292.

Ware, J., Sherbourne, C., 1992. The MOS, 36-item Short-Form Health Survey (SF-36) I: conceptual framework and item selection. Med. Care 30, 473-483.

Wegener, S., Redoblado-Hodge, M.A., Wegener, S., Lucas, S., Fitzgerald, D., Harris, A., Brennan, J., 2005. Relative contributions of psychiatric symptoms and neuropsychological functioning to quality of life in first-episode psychosis. Aust. N. Z. J. Psychiatry $39,487-492$.

World Health Organization, 1998. Development of the World Health Organization WHOQOL-BREF quality of life assessment. The WHOQOL Group. Psychol. Med. 28, 551-558.

Xiang, T.Y., Weng, Y.Z., Leung, C.M., Tang, W.K., Ungvari, G.S., 2007. Quality of life of Chinese schizophrenia outpatients in Hong Kong: relationship to sociodemographic factors and symptomatology. Aust. N. Z. J. Psychiatry 41, 442-449.

Xiang, T.Y., Weng, Y.Z., Leung, C.M., Tang, W.K., Ungvari, G.S., 2008. Subjective quality of life in outpatients with schizophrenia in Hong Kong and Beijing: relationship with sociodemographic and clinical factors. Qual. Life Res. 17, 27-36.

Xiang, T.Y., Chiu, H.F.K., Ungvari, G.S., 2010. Quality of life and mental health in Chinese culture. Curr. Opin. Psychiatry 23, 43-47. 